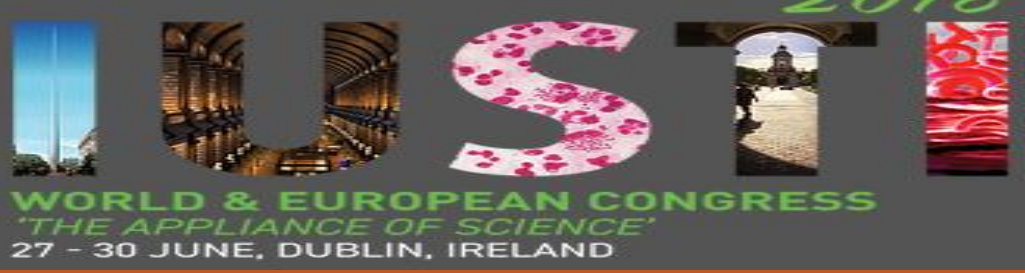

\title{
HIV Prevalence among men who have sex with men in Paraguay ,2017
}

Ríos P. ${ }^{1,}$ Aguilar G. ${ }^{1,2}$; Estigarribia G. ${ }^{1,2}$, Samudio T, Kawabata A. ${ }^{2}$, Ortiz A. ${ }^{1 .}$

Regional Health Research Institute, National University of Caaguazú1: National HIV program².

Background: High HIV/syphilis prevalence have been reported in men who have sex with men (MSM) all over the world. MSM always play a key role in the global HIV epidemic.In Paraguay, 38\% of men diagnosed annually are MSM. In this context, is important to emphasize the risk behavior studies of the MSM in order to have information that allows to redirect the interventions and thus to reduce new infections and meet the goals of the Sustainable Development Goals. Our goal in this study was to estimate the HIV prevalence and Sexual behavior among MSM in Paraguay at 2017.
HIV/Syphilis prevalence, Condom use, Access to HIV testing

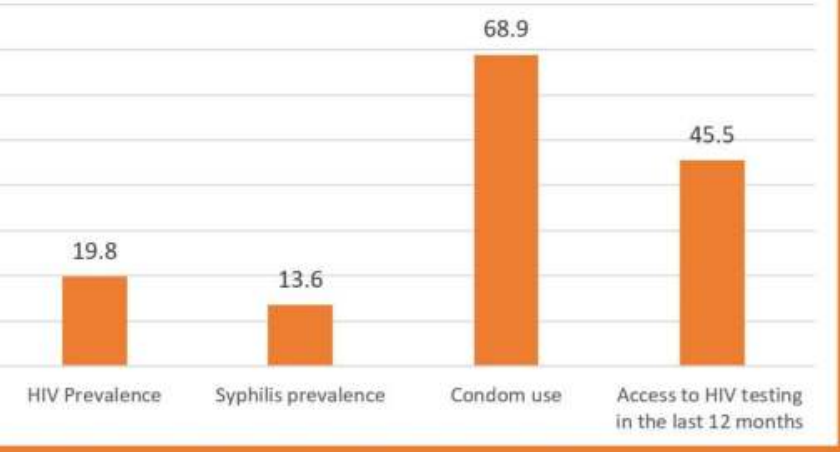

Results: A total of 750 MSM were enrolled in the survey. The majority of the MSM (62\%) were less than 25 years old, (68.3\%) identified himself as gay. The HIV prevalence was $19.8 \%$ (95\% confidence interval:16.3-23.3) and the syphilis prevalence was $13.6 \%$ (95\% confidence interval ;10.1-17.0). Condom use was $68.9 \%$ (95\% confidence interval: 64.6-73.2). Access to HIV testing in the last 12 months was $45.5 \%(95 \%$ confidence interval ;37.3\%-53.6\%)-

Conclusion: The prevalence of HIV and syphilis was high among MSM in Paraguay. Prevention strategies should focus on hsh, gay, and differentiated strategies in hidden MSMs to improve condom use and access to HIV testing.
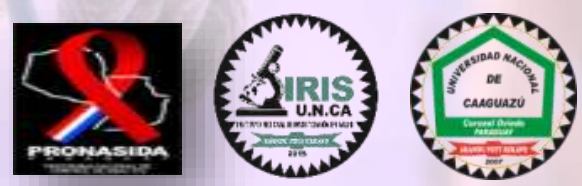